7. Child Lang. 44 (2017), 719-733. (C) Cambridge University Press 2016

This is an Open Access article, distributed under the terms of the Creative Commons

Attribution licence (http://creativecommons.org/licenses/by/4.o/), which permits unrestricted re-use, distribution, and reproduction in any medium, provided the original work is properly cited.

doi:10.1017/So305000916000076

BRIEF RESEARCH REPORT

\title{
Do acting out verbs with dolls and comparison learning between scenes boost toddlers' verb comprehension?*
}

\author{
AMY LOUISE SCHWARZ \\ Texas State University \\ ANNE VAN KLEECK \\ The University of Texas at Dallas \\ MANDY J. MAGUIRE \\ The University of Texas at Dallas
}

A ND

HERVÉ ABDI

The University of Texas at Dallas

(Received 26 November 2013-Revised 23 August 2014-Accepted I 5 Fanuary 2016 First published online I5 March 20I6)

ABSTRACT

To better understand how toddlers integrate multiple learning strategies to acquire verbs, we compared sensorimotor recruitment and comparison learning because both strategies are thought to boost

\footnotetext{
* This research was supported by a New Century Doctoral Scholarship awarded to the first author by the American Speech Language and Hearing Foundation. We give special thanks to Dr Christine Dollaghan, Dr Janie Childers, and Dr Raúl Rojas for their suggestions on this project's design. Hervé Abdi would like to acknowledge the support of an EURIAS fellowship at the Paris Institute for Advanced Studies (France), with the support of the European Union's 7 th Framework Program for research, and from funding from the French state managed by the Agence Nationale de la Recherche (program: Investissements d'Avenir, ANR-II-LABX-0027- or Labex RFIEA+). Address for correspondence: Amy Louise Schwarz, Assistant Professor, Department of Communication Disorders, Texas State University, Health Professions Building, Room ı69, San Marcos, Texas 78666; e-mail: als2 I7@txstate.edu.
} 
children's access to scene-level information. For sensorimotor recruitment, we tested having toddlers use dolls as agents and compared this strategy with having toddlers observe another person enact verbs with dolls. For comparison learning, we compared providing pairs of: (a) training scenes in which animate objects with similar body-shapes maintained agent/patient roles with (b) scenes in which objects with dissimilar body-shapes switched agent/patient roles. Only comparison learning boosted verb comprehension.

\section{INTRODUCTION}

To learn verbs, toddlers use multiple learning strategies (e.g. Gillette, Gleitman, Gleitman \& Lederer, I 999; James \& Maouene, 2009; Maouene, Hidaka \& Smith, 2008; Yuan, Fisher \& Snedeker, 2012). SENSORIMOTOR RECruitment (e.g. Huttenlocher, Smiley \& Charney, ig83) and COMPARISON LEARNing (e.g. Christie \& Gentner, 20Io, 20I4) are two learning strategies thought to boost children's access to scene-level information. Sensorimotor recruitment is grounded in embodied cognition - a set of theories explaining how cognitive processes are built upon sensorimotor processes (e.g. Laakso, 20 I I). Comparison learning is grounded in analogical reasoning: it holds that humans naturally compare scenes to identify the relational concept shared by the scenes (e.g. Christie \& Gentner, 2010, 2014). In this study, we tested whether these two learning strategies increased toddlers' ability to map unfamiliar verbs onto actions.

The question we asked is both theoretical and practical: When toddlers use familiar dolls as agents within familiar social routines, will sensorimotor recruitment and comparison learning boost toddlers' verb learning, and if so, do toddlers rely on these strategies equally? Knowing the relative importance of these strategies will help clarify the relationship between sensorimotor recruitment and comparison learning when toddlers learn verbs. It will also highlight strategies an early childhood educator might use to highlight unfamiliar verbs in language activities when manipulatives are used.

From the theoretical perspective of embodied cognition, cognitive processes are grounded in sensorimotor processes (e.g. Laakso, 20 I I). For example, when toddlers use their own bodies to enact the action described by a verb, they better understand the meaning of this verb than when they observe someone else enacting the verb (Huttenlocher et al., 1983). Even though this effect has been empirically established, studies are needed to evaluate language processing in new embodied contexts in order to evaluate embodied cognition's theoretical and practical relevance (Wellsby \& Pexman, 2014). Activity-based learning research offers a continuum of 
agency contexts in which to test how embodied cognition impacts verb learning, including the agency context of children using dolls as agents (Biazak, Marley \& Levin, 2010). Here, we summarize verb processing research when children assume the agent's role and language processing research when children use doll-agents.

James and Swain's (20II) verb training study compared enactment to observation, and established a causal link between neural motor recruitment and verb learning. To control for comprehension, children were taught novel verbs before the experiment began. During training, children enacted one verb set and observed the examiner enact another verb set. During $f$ MRI scans at test, children showed significant neural motor recruitment (as measured from the Blood Oxygen Level Dependent signal) when they heard verbs they had enacted compared to verbs they had watched the examiner enact - a pattern suggesting that enacting verbs helped children understand verbs. This conclusion gains further support from a longitudinal study beginning when children produced single words (Huttenlocher et al., I983). In this study, children were tested on their ability to enact verbs upon request compared to their ability to select videos of people enacting the same verbs. Children at I;8 (years;months) and 2;2 demonstrated significantly better verb comprehension when they enacted verbs than when they observed others enact verbs (Huttenlocher et al., I983).

Researchers have had children use doll-agents and other toys from thematic play sets to test whether enacting scenes enhances reading and listening comprehension (e.g. Biazak et al., 2010; Glenberg, Gutierrez, Levin, Japuntich \& Kaschak, 2004). These studies suggest a continuum for the level of support provided by manipulatives (Marley \& Carbonneau, 20I4). The continuum's easy end includes having children physically manipulate objects (such as doll-agents), followed by having children observe others use manipulatives. The continuum's difficult end includes having children imagine enacting scenes followed by having them NOT engage with manipulatives. For example, in Biazak et al.'s (2010) listening comprehension study, preschoolers were assigned to one of two groups: one group was trained to enact sentences using doll-agents whereas the other group was asked to think about what was happening in each sentence. At test, preschoolers who used doll-agents recalled significantly more action propositions and familiar action verbs than preschoolers from the other group. Although this study is not about verb acquisition (because it used familiar verbs), it is relevant here because it indicates that sensorimotor recruitment boosts comprehension when children use doll-agents to enact sentences with familiar action verbs. In our study, we compared two learning conditions on the continuum's easy end: (a) having toddlers enact unfamiliar verbs with doll-agents to (b) having them 
observe the examiner enact verbs using doll-agents. Given that sensorimotor recruitment from enacting verbs with doll-agents is more subtle than enacting verbs with one's own body, we wondered whether toddlers would gain a greater boost from comparison learning than from sensorimotor recruitment.

Comparison learning holds that when children are presented with two separate scenes that contain the same relational concept, they will spontaneously look for similarities between the scenes (e.g. Christie \& Gentner, 20I0, 20I4). The children's level of experience with the relational concept under investigation determines the strategy they use to make the comparison. Children who have experience with the relational concept can isolate the concept held in common between two scenes without relying on the attributes of objects included in the scenes (e.g. Christie \& Gentner, 2010, 20I4; Gentner \& Toupin, I986). By contrast, children with limited experience with the relational concept can only isolate the concept held in common between scenes when the concrete object attributes - such as the body-shape of toy animals-are highly similar and fill the same role or position in both scenes (e.g. Christie \& Gentner, 2010, 2014; Gentner \& Toupin, I986). The surface similarity of objects contained in both scenes helps children with limited experience align the scenes and notice the relational concept common to both scenes. Our interest here concerns one aspect of comparison learning that explains how children with limited experience identify the relational concept between two scenes.

For example, to test whether children use similarity in body-shape and participant role to learn narratives, Gentner and Toupin (1986) tested children aged 5;0-7;0 and 8;0-10;0 using ALIGNED and CROSS-MAPPED training scenes. Children heard an initial story that included a hunter as the hero, a pony as a friend, and a spider as a villain. Then all the children retold the story in different conditions. In the aligned condition, they used sets of objects with similar body-shapes to fill the same participant roles as in the initial story. For example, children used a cowboy as the hero, a zebra as a friend, and a fly as a villain. This condition was the aligned condition because the manipulatives filling each participant role had similar body-shapes as in the initial story. In the cross-mapped condition, children retold the story using objects with DIFFERENT body-shapes in different participant roles from the initial story. For example, children used a fly as the hero, a cowboy as a friend, and a zebra as a villain. As comparison learning predicts, children aged $5 ; 0-7 ; 0$, but not children aged 8;0-ıо; , retold the narrative correctly significantly more often in the aligned condition than in the cross-mapped condition. Although this study is not about verb learning, it is relevant here because it indicates that aligning scenes by body-shape and participant role boosts identification of 
linguistic information for young children. We extended this design to verb learning.

For verb learning, the effect of alignment is not always clear. For example, when Childers (2008) tested whether comparison learning boosted toddlers' verb learning, she did not find significant results. In her study, the events included inanimate objects with one object used as an instrument. Instead of using cross-mapped events to disrupt children's comparison of two events, Childers used LESS ALIGNED scenes that contained a different number of objects than the first scenes and scenes with role reversals. Childers did not report how these less aligned events compared to the cross-mapped events researchers typically use to test comparison learning (e.g. Christie \& Gentner, 20ıо; Gentner \& Toupin, I986; Mix, 2008). Given the difference in event stimuli between Childers' study and how researchers typically test comparison learning, it is unclear whether her non-significant results are due to a design issue or to comparison learning.

Although comparison learning has not been successfully studied in verb learning, researchers in other domains of learning have shown that comparison learning significantly boosts young children's recall of story structure (Gentner \& Toupin, I986) and their identification of spatial locations (Paik \& Mix, 2008), spatial patterns (Kotovosky \& Gentner, I996), and numerical equivalence (Mix, 2008). Given this strong evidence for comparison learning in young children's ability to infer a relational concept held in common between scenes, we wanted to know whether toddlers used comparison learning to acquire unfamiliar verbs.

The purpose of the present study was to test the relative impact of sensorimotor recruitment and comparison learning on toddlers' verb learning. Embodied research in verb learning is limited to the agency context of having children enact verbs as the agents of actions (Huttenlocher et al., I983; James \& Swain, 20I I). To determine the theoretical importance of embodied cognition, we tested the new embodied context of having toddlers enact verbs using doll-agents. Specifically, we wondered whether having toddlers enact verbs with doll-agents during training, as compared to observation, increased their verb comprehension at test. We hypothesized that toddlers who enacted the verbs with doll-agents would demonstrate significantly better comprehension than toddlers who observed the examiner enact the verbs with doll-agents. We also wondered whether comparison learning would boost verb comprehension more than sensorimotor input acquired from this new agency context. We tested whether providing toddlers with training scenes aligned by object-shape and participant role, as compared to scenes cross-mapped by object-shape and participant role, increased verb comprehension at test. We hypothesized that toddlers receiving 
aligned examples would demonstrate significantly better comprehension than toddlers who received cross-mapped examples. We also hypothesized that comparison learning would have a stronger effect on toddlers' verb comprehension than embodied cognition.

\section{METHOD}

We used a between-group design, a teach-test block procedure, and frequency of correct enactments as the outcome measure. We analyzed the first test trial out of four trials of each test block, to make our work comparable with previous research (e.g. Childers, 2008; Maguire, Hirsh-Pasek, Golinkoff \& Brandone, 2008). We used PEEKABOo and I'M-GONNA-GET-YOU as the experimental context because both games have a contingent-response structure, are played between adults and children aged $0 ; 8$ to 2; I979), and are therefore familiar to children aged 2;6. In late occurring variations, children participate in the games and assume either agent or patient roles. Children also play these games alone, substituting toys for the agent/patient roles (Ratner \& Bruner, I978).

To address how much children depend on object-shape when learning verbs, we included two different potential agent shapes: (a) four-legged animals (cow, dog, horse, and zebra) and (b) two-legged human occupation dolls (cowgirl, doctor, fireman, and policeman), the labels for which are found in the MacArthur-Bates Communication Development Index (Fenson, Marchman, Thal, Dale, Reznick \& Bates, 2004). Adults rated doll body-shapes (quadruped, biped) as significantly different ( $p$ $<.0 \circ$ I, Wilcoxon signed rank test). Animal-human occupation doll pairs were quasi-randomized within and across teach-test blocks. The experiment included two props constructed from Lego (a room, a cart) to create the contexts for the two games. The room was the context for hiding in the peekaboo game and the cart was the context for fleeing in the I'm-gonna-get-you game. The examiner pulled the cart using an attached string.

We taught two causal transitive verbs with nonsense labels that were specific kinds of hits (Levin, I993). SwIPE means 'to hit with a swinging motion' (American Heritage Dictionary, 2007) and REAR-BUTT-an invented verb-means to hit using the rear with a pushing motion. Two-legged and four-legged animals exhibit both behaviors. In four-legged mammals, paw and foreleg swiping commonly occurs in social play (Burghardt, 2005) and fighting (Rubenstein \& Hack, I992). We used Storkel and Hoover's (2010) online calculator to verify that swipe and rear-butt were unfamiliar and then to create nonsense labels (swipe $=$ HAPE, rear-butt $=\mathrm{GOKE})$ that controlled for phonotactic features. 
Children were taught the two verbs (hape, goke) in counterbalanced order within two games (peekaboo, I'm-gonna-get-you) whose order was counterbalanced. The doll type (occupation doll, animal doll) used in the agent role within the games was counterbalanced across children. Animalhuman occupation doll pairs were randomized into a unique order for each child. Children received six training trials for each verb immediately followed by four testing trials. We counterbalanced the content of teach-test blocks, which included which verb was taught first, which game occurred first, and which doll type (animal, human) occurred as the agent. The variables of verb, game, block order, and agent doll created eight randomized sets of stimuli. We used a between-group design that included sixteen toddlers in each experimental group. The eight randomized sets of stimuli occurred twice in each experimental group.

We determined how many children to test based on Huttenlocher et al.'s ( 1983$)$ results for children aged 2;2 $\left(r_{\text {contrast }}=46\right)$ and Mix's (2008) results for numerical equivalence in children aged $3 ; 6\left(r_{\text {contrast }}=.7 \mathrm{I}\right)$. We recruited seventy-five children aged 2;4 to 2; I I who were native English speakers and had no neurological, language, or general developmental difficulties based on parent report in the Language Development Survey (LDS; Rescorla \& Achenbach, 2002). Eleven children (14.67\%) were excluded because they did not complete the experiment $(6 /$ I I ) or because the examiner erred during the procedure (5/I I), leaving sixty-four children $\left(M_{\text {age }}=2 ; 7, S D=0 ; 2,50 \%\right.$ female). Parents reported $87 \cdot 89 \%$ knew the doll names, $98 \cdot 44 \%$ knew both games, and $97 \cdot 79 \%$ produced the four consonant sounds in the verbs. We randomly assigned children to the four training groups shown in Table I based on their $L D S$ scores, which were not significantly different $(p=\cdot 90$, Kruskal-Wallis test).

The procedure took approximately i 5 minutes. The 'Appendix' includes the training and testing scripts. In the warm-up activity, the examiner handed the child each doll and labeled it. Then, she twice encouraged the child to enact actions with an animal-human occupation doll pair during a free-play period by asking: "What do they do together?" The examiner encouraged the child to enact actions with animal-human occupation doll pairs during the same action sequence that was in each game's test trials. During piloting of the warm-up, we identified the default actions of up-and-down stomping, body slamming, and head-butting.

Training trials were presented in pairs to invite comparison. We used three animal-human occupation doll pairs during each set of six training trials, with the same doll pairs used in two successive trials. For children assigned to enact verbs, the examiner enacted the first trial in each pair and children enacted the second trial with the same doll pair. For children assigned to observe, the examiner enacted both trials. There was no break between paired trials. There was a brief break after each pair of trials 
TABLE I. Frequency of enactments across two test trials

\begin{tabular}{lcc}
\hline & Incorrect & Correct \\
\hline Children use dolls in aligned scenes & 7 & 25 \\
Children observe the examiner use dolls in aligned scenes & IO & 22 \\
Children use dolls in cross-mapped scenes & 23 & 9 \\
Children observe the examiner use dolls in cross-mapped scenes & 24 & 8 \\
\hline
\end{tabular}

while the examiner selected two new dolls for the next two trials. In the second, fourth, and sixth training trials, the shape of the doll filling the agent role was dictated by whether children were assigned to aligned or cross-mapped training scenes. The four test trials for one verb immediately followed the six training trials for that verb and included the same game.

A correct score for swiping differed by doll type. The human occupation doll's hand had to deliberately tap any part of the animal doll. The animal doll had to be oriented at a 45 to 90 degree angle and its front legs/hooves had to deliberately tap the occupation doll. A correct score for rear-butting was the same for all dolls. The agent doll had to tap the patient doll with its buttocks. Children were credited with a correct response regardless of which doll they used to perform the action.

Students who were blind to the study's purpose scored independently all videotaped test trials. Cohen's kappa for inter-judge reliability on $25 \%$ of the data was .88. Procedural and script fidelity across phases ranged from $94.57 \%$ to $100 \cdot 00 \%$. Scoring of agent/patient doll use for the post-hoc analysis was $96 \cdot 88 \%$.

\section{RESULTS}

Table I shows frequency counts for each experimental group. To test logistic regression's assumptions, we used multiple correspondence analysis from the [R]-based ExPosition package (Beaton, Chin Fatt \& Abdi, 20I4; R Development Core Team, 20I4). From this analysis, we found that the data contained no outliers and that the predictors were not redundant.

None of the potential nuisance variables (within-subject: verb, order in which the verbs were taught, games; between-subject: doll type as agent, eight randomized sets of stimuli) significantly influenced verb comprehension. We generated four fixed-effect models to determine the best fit for the data using the forced entry method (no A PRIORI decisions; Field, Miles \& Field, 20I2). Model I predicted the likelihood of verb comprehension without the contrasting conditions. Model 2 predicted the likelihood of verb comprehension when children enacted verbs using doll-agents contrasted with observation $\left(\chi^{2}(\mathrm{I})=0.50, p=\cdot 48\right)$. Model 3 , the 
best fit model, built on Model 2, adding the contrast of aligned and cross-mapped training events $\left(\chi^{2}(2)=29 \cdot 90, p<.00 \mathrm{I}\right)$. Model 4 built on Model 3, adding the interaction term $\left(\chi^{2}(3)=30.06, p<.00 \mathrm{I}\right)$, which was non-significant $(Z=0 \cdot 40, p=\cdot 69)$. Table 2 shows results for Models I and 3 .

Using the corrected $\alpha_{\text {Sidak }}$ of .025 (i.e. $\alpha$ adjusted for two statistical tests), Model 3 revealed three findings. Having children use doll-agents (vs. observation) was not a significant predictor of toddlers' verb comprehension. Children who observed the examiner use doll-agents in cross-mapped training scenes (the base category) were significantly less likely $(.23)$ than chance $(.50)$ to comprehend verbs. Compared to the base category, children who received aligned scenes and used doll-agents to enact the verbs were very likely $(.89)$ to correctly enact verbs at test.

The social exchange games used in our study have clearly defined agent/ patient roles. A possible explanation for our findings is that by continuously switching the agent/patient roles during training, toddlers receiving cross-mapped scenes might have been confused about which dolls were the agents in the games. To test this possible explanation, we re-scored incorrect data for toddlers who received cross-mapped scenes based on whether they used the agent doll (35/47), patient doll (o/47), ambiguous (0/47), or made no contact between dolls (12/47). The probability that toddlers would use the agent doll in our study was .745 , $95 \% \mathrm{CI}=[.597, .86 \mathrm{I}]$. These findings show that toddlers in the cross-mapped groups used the correct doll-agent, but made action errors when asked to demonstrate the verbs. So, toddlers used the object attribute of body-shape to identify the meanings of the unfamiliar verbs held in common across scenes just as comparison learning predicts.

\section{DISCUSSION}

We compared sensorimotor recruitment and comparison learning to determine their relative contributions to toddlers' verb comprehension. We found that comparison learning boosted toddlers' verb comprehension, but sensorimotor recruitment did not.

Our results indicate that toddlers can use comparison learning to acquire verbs, but require those scene pairs to be aligned by object shapes and participant roles. These data support the claim made by comparison learning: when comparing two scenes that share a relational concept - such as an action expressed by an unfamiliar verb-children who have limited experience with the concept will rely on object attributes and the participant roles they fill to identify the relational concept shared by the two scenes.

What are the practical implications of these findings for early childhood educators who regularly use manipulatives - such as dolls, blocks, trucks, 
TABLE 2. Logistic regression results

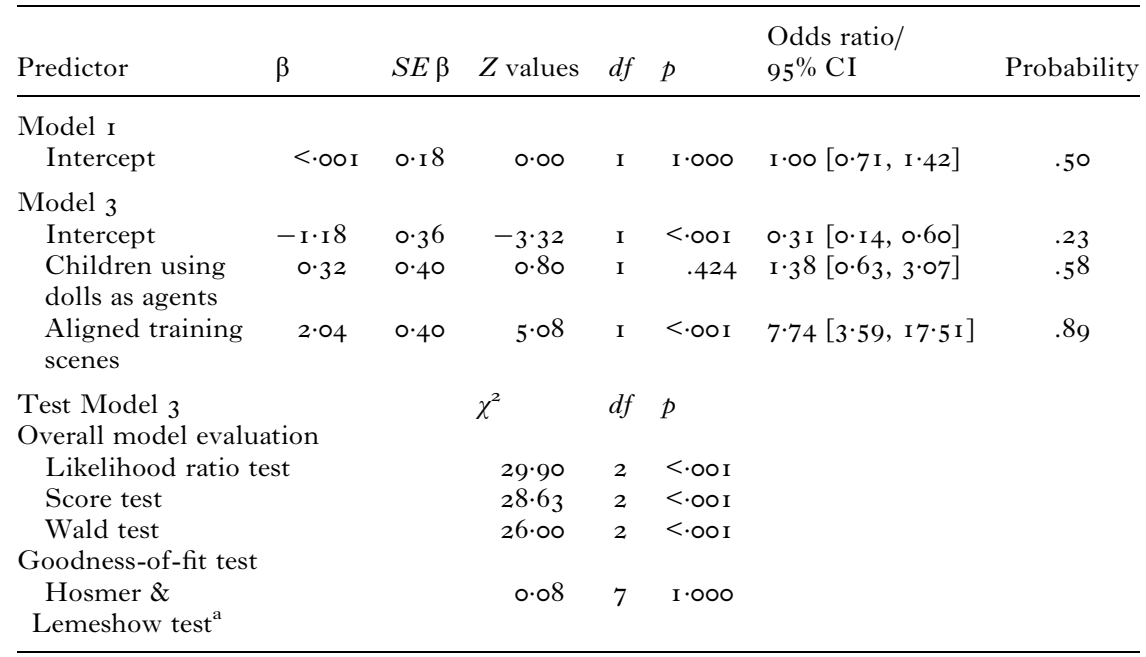

NOTES: [R] functions: glm, anova, wald.test, hoslem.test; ${ }^{a}$ a non-significant finding indicates the model fits the data well.

and Play-Doh-during language activities with toddlers (e.g. Copple \& Bredekamp, 2009; Kontos, I 999; O’Brien \& Bi, I 995)? Our results suggest that to help toddlers learn unfamiliar verbs, early childhood educators can use familiar toys and play routines to create scenarios that include toys aligned by shape and participant role.

An important practical and theoretical issue concerning comparison learning remains: comparison learning posits that once children have sufficient experience with a relational concept-such as an unfamiliar verb - they will identify that concept without relying on the attributes of objects when comparing scenes. We wonder whether our findings are due to the toddlers' inexperience with attending to changing scenes or to their cognitive/linguistic development. In other words, if toddlers had simply had more exposure to cross-mapped examples across a few days, could they have learned to identify the unfamiliar verbs? Or is there an age at which children are cognitively ready to identify unfamiliar verbs without attending to the attributes of objects in the scenes?

We did not find a significant difference in verb comprehension when toddlers enacted verbs with doll-agents compared to observation. This comparison tests a new agency context from the continuum used in the activity-based learning embodiment research, which, so far, had not been applied to verb acquisition. This continuum identifies the different levels of support that manipulatives provide when children learn new concepts. 
Children move from concrete representations that require object manipulation to simulated experiences (Marley \& Carbonneau, 20I4). Our results suggest that children aged 2;6 have sufficient experience with their bodies and with familiar objects to represent unfamiliar verbs by observing actions. Recall that children aged $\mathrm{I} ; 8$ and 2;2 demonstrated significantly better comprehension when they enacted verbs than when they observed others enact verbs (Huttenlocher et al., 1983). Although toddlers in Huttenlocher et al. used themselves as agents instead of using dolls - as toddlers did in our study - the combined results suggest that somewhere between ages 2;2 and 2;6 toddlers have enough experience to acquire verbs through observation alone.

One could object to the assumption underlying activity-based learning studies that having children use doll-agents is an embodied context. Specifically, is there evidence supporting the idea that sensorimotor recruitment occurs when a person uses a substitute agent? Studies using grip force sensors to measure sensorimotor recruitment of verbs describing the behavior of a third person offer an analogous situation to children using doll-agents. Grip force sensors - flat disks participants hold between thumb and fingers-measure the amount and timing of involuntary pressure during sentence processing (i.e. grip force compression). This situation is analogous to using doll-agents because both involve a substitute agent and both actions are more subtle than might be experienced if one were to assume the agent's role when enacting verbs. Aravena et al. (2014, p. 3) compared grip force compression when adults heard three types of sentences: (a) those containing action verbs as the focus of the sentence (At the gym, Fiona lifts the dumbbell), (b) those containing volitional verbs as the focus (In the plane, Laure wants to lift her luggage), and (c) those containing nouns as the focus (In the spring, Edmonde loves the flower-bush in her garden). Adults produced significantly greater grip force compression when they heard sentences focused on action verbs than when they heard sentences focused on volitional verbs $(p=.02, d=0.92)$ or sentences focused on nouns $(p=.007, d=\mathrm{I} \cdot 26)$. Aravena et al. concluded that action-focused sentences induce sensorimotor activation. Assuming toddlers experience similar patterns of sensorimotor activation when they use manipulatives, our findings suggest that this activation does not help toddlers acquire unfamiliar verbs.

In conclusion, children aged 2;6 receive a significant boost in verb comprehension from comparison learning, but not from sensorimotor recruitment. Is there an earlier time in development when sensorimotor recruitment might significantly boost verb comprehension more than comparison learning? Effect sizes calculated from Huttenlocher et al. (1983) suggest that this time might occur sometime before children are I;8. By comparing how these two strategies and others impact verb 
learning at different points in development, we can begin to understand how children weight different learning strategies over time when acquiring verbs.

\section{REFERENCES}

American heritage dictionary of the English language (2007). 4th ed. Boston, MA: Houghton Mifflin.

Aravena, P., Courson, M., Frak, V., Cheylus, A., Paulignan, Y., Deprez, V. \& Nazir, T. (20I4). Action relevance in linguistic context drives word-induced motor activity. Frontiers in Human Neuroscience 8, I-I 2.

Beaton, D., Chin Fatt, C. R. \& Abdi, H. (20I4). An ExPosition of multivariate analysis with the singular value decomposition in R. Computational Statistics $\mathcal{\sigma}^{\circ}$ Data Analysis 72, 1 76-189.

Biazak, J. E., Marley, S. C. \& Levin, J. R. (2010). Does an activity-based learning strategy improve preschool children's memory for narrative passages? Early Childhood Research Quarterly 25, 515-26.

Bruner, J. \& Sherwood, V. (1976). Peekaboo and the learning of rule structure. In J. Bruner, A. Jolly \& K. Sylva (eds), Play: its role in evolution and development (pp. 277-285). New York, NY: Basic Books.

Burghardt, G. (2005). Play in the placental mammals. In The genesis of animal play: testing the limits (pp. I $9 \mathrm{I}-2 \mathrm{I} 2$ ). Cambridge, MA: MIT Press.

Childers, J. B. (2008). The structural alignment and comparison of events in verb acquisition. In V. S. Sloutsky, B. C. Love \& K. McRae (eds), Proceedings of the 3oth Annual Cognitive Science Society (pp. 68I-686). Austin, TX: Cognitive Science Society.

Christie, S. \& Gentner, D. (2010). Where hypotheses come from: learning new relations by structural alignment. Fournal of Cognition and Development II, $356-73$.

Christie, S. \& Gentner, D. (20I4). Language helps children succeed on a classic analogy task. Cognitive Science 38, 383-97.

Copple, C. \& Bredekamp, S. (2009). Developmentally appropriate practice. Washington, DC: National Association for the Education of Young Children.

Fenson, L., Marchman, V. A., Thal, D. J., Dale, P. S., Reznick, J. S. \& Bates, E. (2004). MacArthur-Bates Communicative Development Inventories. Baltimore, MD: Paul H. Brookes Publishing Co.

Field, A., Miles, J. \& Field, Z. (2012). Discovering statistics using R. Los Angeles: Sage.

Gentner, D. \& Toupin. (I 986 ). Systematicity and surface similarity in the development of analogy. Cognitive Science Io, 277-300.

Gillette, J., Gleitman, H., Gleitman, L. \& Lederer, A. (I999). Human simulations of vocabulary learning. Cognition 73, I35-76.

Glenberg, A. M., Gutierrez, T., Levin, J. R., Japuntich, S. \& Kaschak, M. P. (2004). Activity and imagined activity can enhance young children's reading comprehension. Fournal of Educational Psychology 96, 424-36.

Gustafson, G. E., Green, J. A. \& West, M. J. (1979). The infant's changing role in motherinfant games: the growth of social skills. Infant Behavior and Development 2, 30 I-8.

Huttenlocher, J., Smiley, P. \& Charney, R. (1983). Emergence of action categories in the child: evidence from verb meanings. Psychological Review 9o, 72-93.

James, K. H. \& Maouene, J. (2009). Auditory verb perception recruits motor systems in the developing brain: an $f$ MRI investigation. Developmental Science 12, F26-34.

James, K. H. \& Swain, S. (20I I). Only self-generated actions create sensori-motor systems in the developing brain. Developmental Science 14, 673-8.

Kontos, S. (I 999). Preschool teachers' talk, roles, and activity settings during free play. Early Childhood Research Quarterly 14, 363-82.

Kotovsky, L. \& Gentner, D. (r996). Comparison and categorization in the development of relational similarity. Child Development 67, 2797-2822.

Laakso, A. (20I I). Embodiment and development in cognitive science. Cognition, Brain $\xi^{\circ}$ Behavior: An Interdisciplinary fournal 15, 409-25. 
Levin, B. (1993). English verb classes and alternations. Chicago, IL: University of Chicago Press.

Maguire, M. J., Hirsh-Pasek, K., Golinkoff, R. M. \& Brandone, A. C. (2008). Focusing on the relation: fewer exemplars facilitate children's initial verb learning and extension. Developmental Science II, 628-34.

Maouene, J., Hidaka, S. \& Smith, L. B. (2008). Body parts and early-learned verbs. Cognitive Science 32, I200-16.

Marley, S. C. \& Carbonneau, K. J. (20I4). Future directions for theory and research with instructional manipulatives: commentary on special issue papers. Educational Psychology Review 26, I-7.

Mix, K. S. (2008). Children's equivalence judgements: crossmapping effects. Cognitive Development 23, I9I-203.

O'Brien, M. \& Bi, X. (I995). Language learning in context: teacher and toddler speech in three classroom play areas. Topics in Early Childhood Special Education 15, I $48-63$.

Paik, J. H. \& Mix, K. S. (2008). It's all relative: different levels of relational similarity used in children's comparisons. British Fournal of Developmental Psychology 26, 499-505.

$\mathrm{R}$ Development Core Team. (2014). $R$ : a language and environment for statistical computing. Vienna: R Foundation for Statistical Computing.

Ratner, N. \& Bruner, J. (1978). Games, social exchange and the acquisition of language. Fournal of Child Language 5, 391-40 I.

Rescorla, L. \& Achenbach, T. M. (2002). Use of language development survey (LDS) in a national probability sample of children i8 to 35 months old. Fournal of Speech, Language, and Hearing Research 45, 733-43.

Rubenstein, D. I. \& Hack, M. A. (I992). Horse signals: the sounds and scents of fury. Evolutionary Ecology 6, 254-60.

Storkel, H. L. \& Hoover, J. R. (2010). An on-line calculator to compute phonotactic probability and neighborhood density based on child corpora of spoken American English. Behavioral Research Methods 42, 497-506.

Wellsby, M. \& Pexman, P. M. (2014). Developing embodied cognition: insights from children's concepts and language processing. Frontiers in Human Neuroscience 5, I-10.

Yuan, S., Fisher, C. \& Snedeker, J. (2012). Counting the nouns: simple structural cues to verb meaning. Child Development 83, I382-99.

\section{Appendix}

This appendix includes the complete training script for one training trial, an explanation of how the training script changed for the second training trial, and the testing scripts for both social exchange games.

\section{PEEKABOO SCRIPT}

\section{First training trial in a pair of trials}

"Look the fireman and the cow are gonna play peekaboo. The fireman's gonna hide this time. Then the cow's gonna <goke/hape> him to make him come out."

(The fireman hides in the Lego room facing the window.)

"Okay. The fireman's hidden. The cow's gonna < goke/hape> the fireman to make him come out."

(The cow approaches the hiding fireman.)

"He's < goking/haping> him. He's < goking/haping> him." 
(The cow performs the action on the fireman twice. The fireman turns around and says)

"Peekaboo. I came out because you < goked/haped $>$ me."

“They're going to play again. It's the < doll name's $>$ turn. He's gonna hide this time."

\section{Second training trial in a pair of trials}

For children who observed the examiner use dolls as agents in aligned events, the examiner repeated the script for the first training trial. For children who observed the examiner use dolls as agents in cross-mapped events, the examiner repeated the script for the first training trial but switched the dolls filling the agent/patient roles. Children who enacted verbs using dolls as agents in the aligned training events and children who enacted verbs using dolls as agents in cross-mapped training events enacted the verbs in the second training trial. If a child in these latter two groups did not enact the verb during the training trial, the examiner provided a physical prompt. The examiner reached across the table, oriented the doll correctly in the child's hand holding the doll so that the doll filling the agent role correctly acted upon (swiping or rear-butting) the doll filling the patient role.

\section{One test trial}

“It's your turn. You're the dog. Look. The doctor is gonna hide this time. The dog's gonna < goke/hape> him to make him come out."

(The doctor hides in the Lego room facing the window.)

"Okay. The doctor's hidden. The dog's gonna < goke/hape> the doctor to make him come out. Show me. The dog is <goking/haping > the doctor." (If the child does not respond within a count of three 'Mississippis', the examiner says)

"Can you do it? Can you show me he's <goking/haping > the doctor?"

(If the child demonstrates the correct action within a count of ten 'Mississippis', the doctor turns around and exclaims)

"Peekaboo!"

(If the child does not demonstrate the correct action within the count, the examiner takes the child's doll, signaling the test trial's end.)

\section{I'M-GONNA-GET-YOU SCRIPT}

\section{First training trial in a pair of trials}

"Look the horse and the policeman are gonna play I'm-gonna-get-you. The horse is gonna get the policeman. He's gonna <goke/hape $>$ him to make him fall down. The horse is gonna $<$ goke/hape $>$ the policeman so he'll fall down." 
(The examiner puts the policeman in the cart and pulls the cart by a string attached in the front. The horse chases the policeman.)

"He's < goking/haping> him! He's < goking/haping> him!"

(The horse performs the action on the policeman twice. The policeman falls out of the cart, jumps up and says)

"Oh No! You <goked/haped> me. I fell down because you <goked/ haped> me."

"They're going to play again. It's the < doll name's $>$ turn. He's gonna get the <other doll>."

\section{Second training trial in a pair of trials}

See the explanation for the second training trial under the Peekaboo script for details on how this training trial differed across experimental groups.

\section{One test trial}

"It's your turn. You're the zebra. The zebra is gonna get the cowgirl this time. He's gonna <goke/hape> her to make her fall down. Show me. The zebra is <goking/haping> the cowgirl."

(If the child does not respond within a count of three 'Mississippis', the examiner says)

"Can you do it? Can you show me he's <goking/haping> the cowgirl?"

(If the child demonstrates the correct action within a count of ten 'Mississippis', the cowgirl turns around and exclaims)

"Oh No!"

(If the child does not demonstrate the correct action within the count, the examiner takes the child's doll signaling the test trial's end.) 